\title{
PARTIAL PRESSURE ANALYSIS IN SPACE TESTING ${ }^{1}$
}

\author{
Charles R. Tilford \\ National Institute of Standards and Technology \\ Gaithersburg, MD 20899
}

\begin{abstract}
For vacuum-system or test-article analysis it is often desirable to know the species and partial pressures of the vacuum gases. Residual Gas or Partial Pressure Analyzers (PPAs) are commonly used for this purpose. These are mass spectrometer-type instruments, most commonly employing quadrupole filters. These instruments can be extremely useful, but they should be used with caution. Depending on the instrument design, calibration procedures, and conditions of use, measurements made with these instruments can be accurate to within a few percent, or in error by two or more orders of magnitude. Significant sources of error can include relative gas sensitivities that differ from handbook values by an order of magnitude, changes in sensitivity with pressure by as much as two orders of magnitude, changes in sensitivity with time after exposure to chemically active gases, and the dependence of the sensitivity for one gas on the pressures of other gases. However, for most instruments, these errors can be greatly reduced with proper operating procedures and conditions of use. In this paper, data are presented illustrating performance characteristics for different instruments and gases, operating parameters are recommended to minimize some errors, and calibrations procedures are described that can detect and/or correct other errors.
\end{abstract}

\section{INTRODUCTION}

There are many applications where it is desirable or essential to know not just the total vacuum pressure, but the partial pressures of different gas species. Partial pressure analysis is widely used as a general diagnostic of vacuum system performance, and it is essential when testing systems or components that can be damaged by contaminants (e.g. vacuum pump oil, water). For outgassing studies the identification of the gas species and their partial pressures are an invaluable tool in identifying gas sources and determining the possible interactions between the outgassed material and sensitive components on which it might impinge. These measurements can be made using Partial Pressure Analyzers (PPAs), also known as Residual Gas Analyzers (RGAs), which indicate the partial pressures, as a function of molecular weight, of the gases in a vacuum environment. Although originally developed for the qualitative diagnosis of residual gases in ultra high vacuum systems, the growing need for better contamination data and more sophisticated space experiments has encouraged the application of these instruments to quantitative measurements. However, the performance of these instruments varies significantly, depending on design and conditions of use. In the best cases, after proper calibration, they can be accurate to within a few percent; in other cases, they can have orders-of-magnitude errors. The unpredictable performance of some instruments has caused misleading results - frustrating users and probably discouraging the wider use of a potentially valuable instrument.

\footnotetext{
${ }^{1}$ This work was supported in part by the Applied Physics Laboratory of The Johns Hopkins University.
} 
At a minimum, users can make better use of RGA results if they understand the potential problems and errors. It is also possible, with a reasonable effort, to detect and even minimize some of the more egregious errors. This paper discusses some of the observed performance characteristics of RGAs or PPAs, and suggests calibration or testing procedures that can help to detect and minimize undesirable behavior.

\section{INSTRUMENT BASICS}

Residual gas analyzers are compact mass spectrometers that can be attached to vacuum systems as appendage instruments, or, with minor modifications, immersed directly in the vacuum. Generally, they can be baked, are constructed to be compatible with ultra high vacuum systems, and are designed for high sensitivity so that they can detect and analyze low-level residual gases in a vacuum system. They consist of an electron-impact ionizer, a mass filter, extraction electrodes to transfer ions from the ionizer to the mass filter, and an ion detector at the far end of the mass filter. Ion source designs are varied and can have a significant effect on instrument performance. In the simplest cases they resemble the filament and grid structure of a Bayard-Alpert ionization gage. In some instruments they are "closed" and may be differentially pumped so that the instrument operates at a reduced pressure and samples higher-pressure gases through a restricted conductance. A number of different mass filter types have been used and many of the early instruments used magnetic-sector analyzers. However, most modern commercial RGAs use quadrupole mass filters. In a quadrupole filter a combination of DC and megahertz-range radio frequency voltages impressed on the quadrupole rods increases the probability that ions with a selected mass-tocharge ratio will travel down the axis of the filter structure to the detector; the transmission probability for other ions is much smaller and they are deflected to the rods or the surrounding structure. The detector may be a Faraday cup or, for increased sensitivity, a secondary electron multiplier (SEM). The SEM may be used as an analog current amplifier, or combined with a fast-rise amplifier for ion counting.

Mass ranges for different instruments vary, but ranges of 50 to 200 mass-to-charge ratio are typical. The high-sensitivity design and small size limits the mass resolution of these instruments and it is often necessary to use cracking patterns to distinguish between molecules with small mass differences, e.g., $\mathrm{N}_{2}$ and $\mathrm{CO}$. The minimum-detectable partial pressure is determined by several factors, and ranges from $10^{-14}$ to $10^{-8} \mathrm{~Pa}\left(10^{-16}\right.$ to $10^{-10}$ torr; 1 torr $\left.=133.3 \mathrm{~Pa}\right)$ for different instruments. The ion sources of these instruments typically can operate in pressures as high as $0.1 \mathrm{~Pa}$, however, as will be shown, significant high-pressure nonlinearities can occur in some instruments at pressures of $10^{-3} \mathrm{~Pa}$ or lower. Differential pumping can extend the effective range of the instruments higher, but attention must be paid that sampling problems do not distort the partial-pressure spectrum.

\section{INSTRUMENT PERFORMANCE}

The increased interest in using RGAs for quantitative analysis has prompted several systematic studies of their performance in recent years [Refs. 1-6]. The studies at the National Institute of Standards and Technology (NIST) [Refs. 5,6] have involved the repeated calibrations of more than a dozen different RGAs with partial pressures of different gases between $10^{-7}$ and $10^{-1} \mathrm{~Pa}$. The performance of these instruments, especially mass resolution and sensitivity as a function of mass-to-charge ratio, will depend on a number of factors, including, ion energy, electron emission current, and both the dc and rf potentials in the quadrupole filter. In many instruments these factors can be varied by adjusting different instrument operating parameters. Unfortunately, most manufacturers provide little or no information on the overall performance effects of varying these parameters. Therefore, for some instruments these calibrations were 
repeated with different combinations of instrument operating parameters. In some cases the calibrations were performed with pure gases, in others with combinations of two or three gases, each of which could be independently controlled and measured. The calibrations for inert gases were referenced to NIST primary vacuum standards [Ref. 7], either directly or with calibrated transfer gages. Water calibrations used a new NIST primary water vapor standard [Refs. 6, 8]. Examples of the NIST results are presented below. Further details and results obtained by other workers can be obtained from the references.

\section{Relative Sensitivities}

The sensitivities of ionization gages to different gases are generally presented as "relative sensitivities" - the ratio of the sensitivity for a specific gas to the sensitivity of the same gage for a reference gas, generally nitrogen or argon. Generally, for a given gas the relative sensitivities for different ionization gages do not vary by more than $10 \%$. It is often assumed, both by users and some instrument manufacturers, that these same relative sensitivities can be used for RGAs. However, while the relative sensitivity of an ion gage is determined primarily by the ionization probability of the different gases, the relative sensitivities of RGAs are also strongly influenced by the mass-dependent transmission efficiency of the mass filter, which depends on instrument design and operating parameters. Data are presented in the references showing relative sensitivities for different instruments, operated with Faraday-cup detectors, that differ by as much as a factor of five from the values found for ionization gages, and in at least one case the sensitivity of a particular instrument for helium, relative to argon, could be varied by a factor of 10 simply by changing instrument operating parameters. The sensitivity of SEMs is also mass dependent, and their use will introduce additional variability in the relative sensitivities.

\section{Sensitivity as a Function of Pressure}

As a first approximation, the sensitivity of a hot-cathode ionization-type instrument is independent of pressure, i.e., the ion current is linear with pressure. However, with the more complicated RGAs the linearity can be strongly influenced by instrument design, operating parameters [Ref. 5], and even history of use [Ref. 2]. The most influential operating parameter is the ion-extraction voltage (labeled "ion energy" in many instruments), the potential difference between the center of the quadrupole filter and the anode of the ion source. The observed nonlinearities can be broadly categorized as "low-pressure", typically occurring at pressures of $10^{-4} \mathrm{~Pa}$ and below during operation with high ion-extraction voltage settings, and "high-pressure", typically occurring at pressures of $10^{-3} \mathrm{~Pa}$ and above during operation with low ion-extraction voltages.

The range of observed performance can be appreciated from Fig. 1. The data in this figure, discussed in detail in [Ref. 5], were selected from 27 different data sets obtained for each instrument with different ion-source operating parameters: emission current, electron-accelerating voltage, and ion-extraction voltage. To emphasize the changes in linearity, the data from each set have been normalized to a value of 1 at $10^{-4}$ $\mathrm{Pa}$. The data presented were chosen to illustrate extremes of behavior: best linearity, maximum lowpressure nonlinearity, and maximum high-pressure nonlinearity. For PPA-D the performance illustrated by the line with no symbols (sensitivity constant to within a few percent up to $10^{-2} \mathrm{~Pa}$ ) was obtained for a wide range of operating parameters, the high-pressure nonlinearity was observed only under extreme settings of the operating parameters, and virtually no low-pressure nonlinearity was observed. For PPA-A 
significant nonlinearities were observed for all combinations of operating parameters. The line with no symbols was the best observed performance, and large low- and high-pressure nonlinearities were observed for a wide range of operating parameters. In particular, when operated at low ion-extraction voltages the sensitivity of this instrument changed by more than two orders of magnitude as a function of pressure.

The performance characteristics illustrated in Fig. 1 clearly depend in part on the design of the instrument, but the question remains, how well do these characteristics repeat from one unit to another of the same design? We have carried out brief testing of two additional units of instrument type $A$, and have found similar results to those illustrated in Fig. 1. We have carried out more extensive tests of three additional units of type $D$, and some of the results are illustrated in Fig. 2 . In this test, the three additional units, D-2, D-3, and D-4, were operated with the same operating parameters $(1 \mathrm{~mA}$ emission current, $100 \mathrm{~V}$ electron energy, and $10 \mathrm{~V}$ ion extraction voltage) found to give the most linear behavior for the original instrument, now labelled D-1. As can be seen, all four instruments exhibit very good linearity at low pressures, but there are significant differences for pressures above $10^{-3} \mathrm{~Pa}$. It is quite possible that the limit of linear behavior could be extended to higher pressures for D-2 and D-4 by decreasing the ion energy (ion extraction voltage). One can reasonably conclude that instruments of a given manufacturer and model will probably give similar performance under similar conditions of use, but that for best performance individual instruments will have to be fine tuned.

\section{Background Gas Effects}

It is implicitly assumed that an RGA measures the partial pressure of one gas independent of the pressure of other gases. This assumption has been tested using calibration systems in which two or three gases can be simultaneously and independently controlled and measured [Refs. 2, 5]. The assumption is not always valid.

Figure 3 illustrates the results of such a test with two inert gases. The helium responses of several RGAs were simultaneously monitored as a function of the pressure of a second gas - argon. A helium pressure of $1.2 \times 10^{-4} \mathrm{~Pa}$ was maintained constant to within $1 \%$ throughout the experiment. The experiment started with an argon pressure of $8.6 \times 10^{-5} \mathrm{~Pa}$, which was then reduced to "zero" for the time indicated by the horizontal line on the figure, after which the argon pressure was restored. The changes in the helium responses of four instruments are shown; three are quadrupoles and instrument $F$ is a magnetic-sector. As can be seen, the responses of all four instruments were stable with time, and that of instrument $F$ was little affected by the change in the argon pressure. However, the helium sensitivities of the three quadrupoles variously increased or decreased by up to $12 \%$ when the argon was "turned off". Not included in the figure were data for two other quadrupoles tested at the same time; their response was similar to F, i.e., they showed virtually no change in their helium sensitivity.

The results of Fig. 3 illustrate some of the types of responses observed with inert gases for different instruments. In general, the responses depend not only on the particular instrument, but also on the combination of gases. Typically, argon sensitivities are much less affected by changes in a helium pressure. On the other hand, instruments that are unaffected by changes in argon or helium background pressures can be significantly affected by a change in water pressure. Generally, with higher background pressures the sensitivity changes are larger; in some cases we have seen order-of-magnitude changes for background pressure changes in the $10^{-3}$ to $10^{-1} \mathrm{~Pa}$ range. And there is evidence that the characteristics of a particular instrument are affected by condition and duration of use [Ref. 2]. 


\section{Reactive Gas Effects}

In addition to the prompt sensitivity changes illustrated in Fig. 3, reactive gases can cause changes that are slowly reversible. We have observed such changes during and after operation with $\mathrm{O}_{2}, \mathrm{H}_{2} \mathrm{O}, \mathrm{CO}$, $\mathrm{H}_{2}$, and even $\mathrm{CO}_{2}$. Figure 4 illustrates the results of such a test for three different instruments; two quadrupoles and one magnetic-sector $(\mathrm{F})$. In this case an argon pressure of $1.3 \times 10^{-4} \mathrm{~Pa}$ and a helium pressure of $1.4 \times 10^{-4} \mathrm{~Pa}$ were maintained in the chamber. A water pressure of $4.4 \times 10^{-4} \mathrm{~Pa}$ was then "turned on" during the period indicated on the time axis by arrows. Again, the results differ from instrument to instrument. The argon signal of instrument $F$, the magnetic-sector which did not show a change in sensitivity in Fig. 3, does show a "prompt" change in the argon signal when water is added and taken away, as well as a residual shift in the argon signal after the water is "removed" (slow equilibration of the water pressure accounts for the time-response of the instrument signal). Instrument D-4, an instrument that showed no change in the helium sensitivity during the test illustrated in Fig. 3, shows a definite time-dependent change in argon sensitivity throughout the water exposure, but a prompt recovery with a residual offset when the water is removed. Instrument A-2, of the same type as PPA-A in Fig. 1, exhibits a complicated response to the water, and most notably exhibits a long-term recovery of the argon sensitivity after the water is removed.

Again, the data of Fig. 4 are only illustrative. For a number of other instruments we have observed both positive and negative sensitivity changes after exposure to different reactive gases, followed by longtime-constant decays, similar to that shown for A-2, back to their pre-exposure values. This effect will cause a hysteresis in the indicated partial pressures when the pressure of a reactive gas is increased and then decreased. Typically, we have not observed sensitivity changes greater than about $20 \%$, and some instruments seem to be effectively immune to this behavior.

\section{Absolute Sensitivity and Minimum-Detectable Partial Pressure}

As noted before, RGA sensitivities depend on pressure, gas species, and instrument operating parameters. There are also instrument design features that cause significant variations in sensitivity from one instrument to another. For instance, five instruments of different design, operated with Faraday-cup detectors and "nominal" operating parameters, had sensitivities ranging from 2 to $52 \times 10^{-8} \mathrm{~A} / \mathrm{Pa}$ for an argon pressure of $10^{-4} \mathrm{~Pa}$ [Ref. 5]. The factors influencing absolute sensitivities and minimum-detectable partial pressures are discussed in some detail in Ref. 9, but the most important factor is the ion source design and the coupling of the ions from the source to the mass analyzer. For a given electron emission current, the ion production rate can be increased by electrostatically trapping the electrons to increase their path length within the ionizer. The ions must then be extracted from the source and injected into the mass analyzer; the efficiency of this process will depend on field gradients within the source and between the source and the analyzer - the fields between an ion source and a quadrupole analyzer are particularly important.

Unfortunately, using electron trapping to enhance the "brightness" of an ion source makes the source dependent on space charge and susceptible to the previously-discussed nonlinearities with pressure. Of the instruments tested in Ref. 5, the most sensitive was PPA-A of Fig. 1, the least sensitive was PPA-D. The space charge dependence and nonlinearities of some quadrupole instruments can be decreased, and the sensitivity increased, by operating at higher ion energies, but this will decrease the mass resolution, and can cause nonlinearities at lower pressures. 
The minimal-detectable partial pressure will depend on the instrument sensitivity and the ion detection limit. For one-second sampling times the noise limit of high-quality ammeters and cables is about $10^{-16} \mathrm{~A}$, so the detection limit for PPAs with Faraday cup detectors can be in the range of $10^{-11}$ to $10^{-9} \mathrm{~Pa}$. However, not all ammeters are equal, and in some instruments the limit can be two to three orders of magnitude higher. Secondary electron multipliers can increase the ion current by as much as a factor of $10^{7}$, depending on SEM design and operating voltage. However, SEM noise and quantum limits (1 electron/s corresponds to $1.6 \times 10^{-19} \mathrm{~A}$ ) will limit the minimum-detectable partial pressure to $10^{-14}$ to $10^{-12} \mathrm{~Pa}$ range. By using ion counting and longer sampling times this limit can be reduced somewhat. SEMs are also used to achieve improved, but modest detection limits with lower-cost, noisier ammeters.

As with other RGA characteristics, there is often a tradeoff between sensitivity, linearity, and mass discrimination, and the user should choose an instrument to suit the problem. It should also be noted that the detection limit for a given species can be obscured by overlap from strong neighboring peak - this performance characteristic is generally characterized as "abundance sensitivity". This problem will increase as the mass resolution decreases.

\section{Long-Term Stability}

If Faraday-cup detectors are used and operation with active gases is avoided, the sensitivity of some instruments has been observed to change less than $10 \%$ over a period of months. At the other extreme, during this same time the sensitivity of other instruments changed by as much as a factor of five, although after several months operation the sensitivity of these instruments stabilized to within $25 \%$. The gain of an SEMs can exhibit order-of-magnitude changes over periods of weeks, particularly when the SEM is new, so operation with SEMs results in sensitivities that can be significantly less stable than with Faradaycup detection.

\section{CONTAMINATION}

Contaminants generated in RGAs are a particularly serious problem since they cannot be distinguished from system gases and are generally present at relatively high partial pressures because of conductance limitations. Therefore, it is particularly important to use ultra high vacuum techniques in handling and using RGAs and highly desirable that they be baked.

However, even with a clean RGA, apparent contaminants or anomalous species can be generated by reactions between the hot filament and system gases. A notable example is water (Ref. 6). Operation in water of tungsten or thoria-coated filaments, or the high-voltage discharge in a cold-cathode gage, will generate significant quantities of hydrogen, carbon monoxide (which might be mistaken for nitrogen), and carbon dioxide. Variable amounts of oxygen will also be produced, depending on water pressure and time. Detectable amounts of atomic oxygen and $\mathrm{H}_{3} \mathrm{O}$ (hydronium, which can be confused with fluorine) are also produced. Since water is often the dominant gas in a vacuum system, and some space experiments, these reactions can cause the misidentification of species not present, or present in smaller quantities.

\section{RECOMMENDATIONS}

If RGAs are to be used to their full potential it is clearly desirable to avoid or minimize the aberrant behavior that has been observed for some instruments. The most and least desirable instrument characteristics will depend on the application, and there is probably no perfect instrument for all 
applications, although for almost any application it is probably undesirable for the sensitivity for one gas to depend on the pressure of other gases. As noted, there will be tradeoffs between characteristics, and there is not a perfect correlation between price and performance.

It is important that the user decide what mass range, minimum-detectable partial pressure, and mass resolution the application requires. Extending capabilities beyond actual requirements in any of these areas will incur penalties in other aspects of performance, and instrument cost. Having prioritized instrument requirements, three steps should be taken: select an RGA that most closely matches those requirements, adjust the instrument operating parameters to optimize its performance, and calibrate it with a mix of gases and over a range of pressures that approximate the actual conditions of use. This is clearly easier said than done, but not totally impossible.

As a first effort, ask the manufacturer for calibration data; if the RGA readout is in pressure units, demand calibration data. In many cases the manufacturers do not know or may not disclose much of the information of interest, so all three steps will probably require instrument testing and calibration by the user. NIST is currently developing simpler calibration systems of the type described in Ref. 5. These are designed to allow users to calibrate RGAs with several gases at once over a range of pressures. Until such systems are available, RGAs can be calibrated with pure inert gases by comparison with a BayardAlpert (BA) ionization gage (preferably a glass-envelope gage with opposed tungsten filaments) over a wide range of pressures. These ion gages are much more stable and linear than an RGA; with a good controller they will be linear and stable to within $10 \%$ over a wide range of pressures. For better accuracy, a Spinning Rotor Gage can be used (the operation of vacuum gages is discussed in Ref. 10). In general, calibration of the mass scale is not a problem, and it can be quickly checked with respect to common residual gases; e.g., $\mathrm{H}_{2}$ at mass-to-charge ratio of $2, \mathrm{H}_{2} \mathrm{O}$ at 18 and $\mathrm{CO}$ at 28 . Further information on RGA evaluation and calibration procedures is given in Ref. 11.

The reasons why quadrupole instruments behave the way they do are not fully (or even well) understood, but it does appear that space charge in the ion source has a significant influence on the ion extraction process and the instrument sensitivity [Refs. 12,13]. Charge exchange in the ion source can also be important, as well as surface contamination on quadrupole rods [Refs. 2, 14]. Space-charge effects will be enhanced when operating with low ion-extraction voltages. If an increase in the RGA sensitivity at high pressures is observed (e.g., as in Fig. 1), and the instrument allows the adjustment, try operating with higher ion-extraction voltages. It is generally believed that operating with lower emission currents will also reduce high-pressure increases in sensitivity; our experience is that this is true in some, but not all cases. On the other hand, if the sensitivity increases with decreasing pressure below $10^{-4} \mathrm{~Pa}$, try operating with lower ion-extraction voltages. If a significant pressure dependence of the sensitivity still remains, expect that the sensitivities for different gases will depend on the pressures of other gases,

After optimum operating parameters are established, periodically repeat calibrations with one or more pure gases to check for stability. If an SEM is used, periodically compare it to a Faraday cup and adjust the SEM gain or correct the readings accordingly. As a general rule, we obtain the most reliable results with tungsten filaments. Extended operation at higher pressures in hydrocarbon-contaminated systems can deposit insulating films on the quadrupole rods [Ref. 14] and should be avoided.

With some instruments and under some conditions of use, we have found that after calibration we can make measurements with uncertainties of a few percent. With other instruments and/or conditions of use, it is difficult to keep errors below one or two orders of magnitude. At a minimum, users should have some idea which situation they are dealing with and interpret their results accordingly. 


\section{REFERENCES}

1. F. M. Mao, J. M. Yang, W. E. Austin, and J. H. Leck, "Residual gas analyzers and their use in high vacuum systems", Vacuum $\underline{37}$, 335-8 (1987).

2. Fu Ming Mao and J. H. Leck, "The quadrupole mass spectrometer in practical operation", Vacuum 37, 669-75 (1987).

3. R. J. Reid and A. P. James, "Characterization of a multiple head residual gas analyzer installation on an electron storage ring", Vacuum $37,339-42$ (1987).

4. J. D. Sankey and A. H. Bass, "A preliminary attempt at the calibration of a small quadrupole mass spectrometer in the ultra-high vacuum portion of its range", Vacuum $\underline{40}$ 309-312 (1990).

5. L. Lieszkovszky, A. R. Filippelli, and C. R. Tilford, "Metrological characteristics of partial pressure analyzers", J. Vac. Sci. Technol. A $\underline{8}$ 3838-3854 (1990).

6. C.R. Tilford, "Characteristics of Partial Pressure Analyzers", in James B. Breckinridge and Alexander J. Marker III (eds.), SPIE Vol. 1761, Damage to Space Optics and Properties and Characteristics of Optical Glass, Vol. 1761, SPIE, Bellingham WA, 1992, p. 119-129.

7. C. R. Tilford, S. Dittmann, and K. E. McCulloh, "The National Bureau of Standards Primary HighVacuum Standard", J. Vac. Sci. Technol.A 6 2853, (1988).

8. S.A. Tison and C.R. Tilford, "Low-Density Water Vapor Measurements; The NIST Primary Standard and Instrument Response", in Benjamin A. Moore and Joseph A. Carpenter, Jr. (eds.), RL/NIST Workshop on Moisture Measurement and Control for Microelectronics, NISTIR 5241, NIST, U.S.A., 1993, p. 19-29.

9. C.R. Tilford, "The Measurement of Very-Low Partial Pressures", J. Vac. Soc. Japan 37 no.7 (1994).

10. Charles R. Tilford, "Pressure and Vacuum Measurements", Chapter 2, Volume VI, Physical Methods of Chemistry, edited by Bryant W. Rossiter, John F. Hamilton, and Roger C. Baetzold, Interscience, New York, (1992).

11. J.A. Basford, M.D. Boeckmann, R.E. Ellefson, A.R. Filippelli, D.H. Hokeboer, L. Lieszkovszky and C.M. Stupak, "Recommended Practice for the Calibration of Mass Spectrometers for Partial Pressure Analysis", J. Vac. Sci. Technol. A 11 no. 3, pg. A22 (1993).

12 M.C. Cowen, W. Allison and J.H. Batey, "Electron space charge effects in ion sources for residual gas analysis", Measurement Sci. Technol. 4, 72 (1993).

13. M.C. Cowen, W. Allison and J.H. Batey, "Nonlinearities in sensitivity of quadrupole partial pressure analyzers operating at higher gas pressures", J. Vac. Sci. Technol. A $\underline{12}, 228$ (1994).

14. W.E. Austin and J.H. Leck, "Optimization of the operation of the small quadrupole mass spectrometer to give minimum long-term instability", Vacuum 41, 2001 (1990). 


\section{FIGURE CAPTIONS}

1. Normalized argon sensitivities for two different quadrupole RGAs (PPA types A and D) operated with different combinations of ion source parameters. The line without symbols illustrates the most linear behavior for each instrument, while the circles and squares illustrate extremes of high- and lowpressure nonlinearities.

2. Normalized argon sensitivities for four different RGAs, all of the same manufacturer and model (type D of Fig. 1), and all operated with the same emission current, electron energy, and ion extraction voltage.

3. Changes in the response of four different instruments to a helium pressure of $1.2 \times 10^{-4} \mathrm{~Pa}$ when an argon pressure of $8.6 \times 10^{-5} \mathrm{~Pa}$ is reduced to zero ("Ar Off) and then restored.

4. Changes in the response of three different instruments to an argon pressure of $1.3 \times 10^{-4} \mathrm{~Pa}$ when a water pressure of $4.4 \times 10^{-4} \mathrm{~Pa}$ is introduced and then removed. 

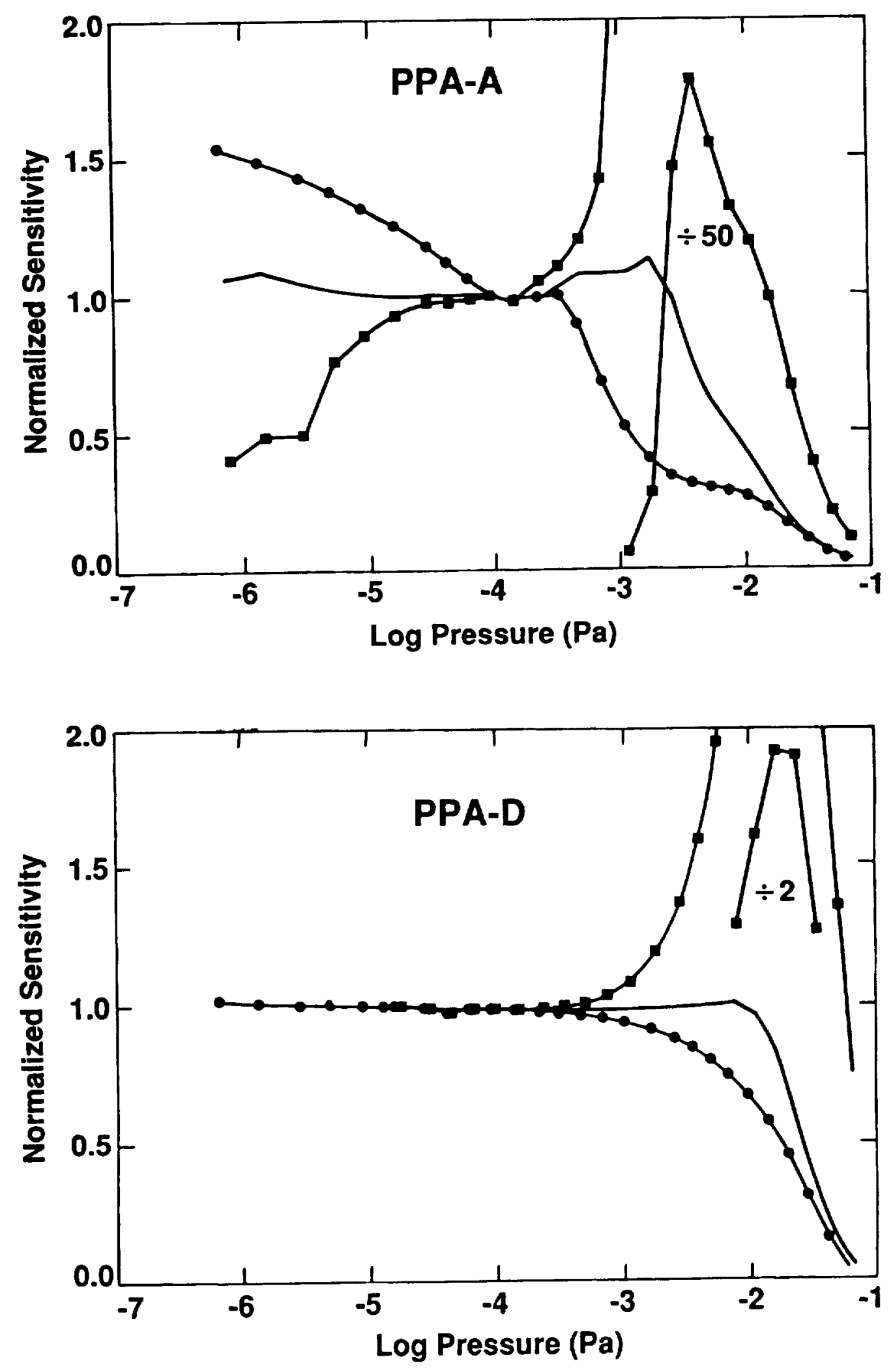


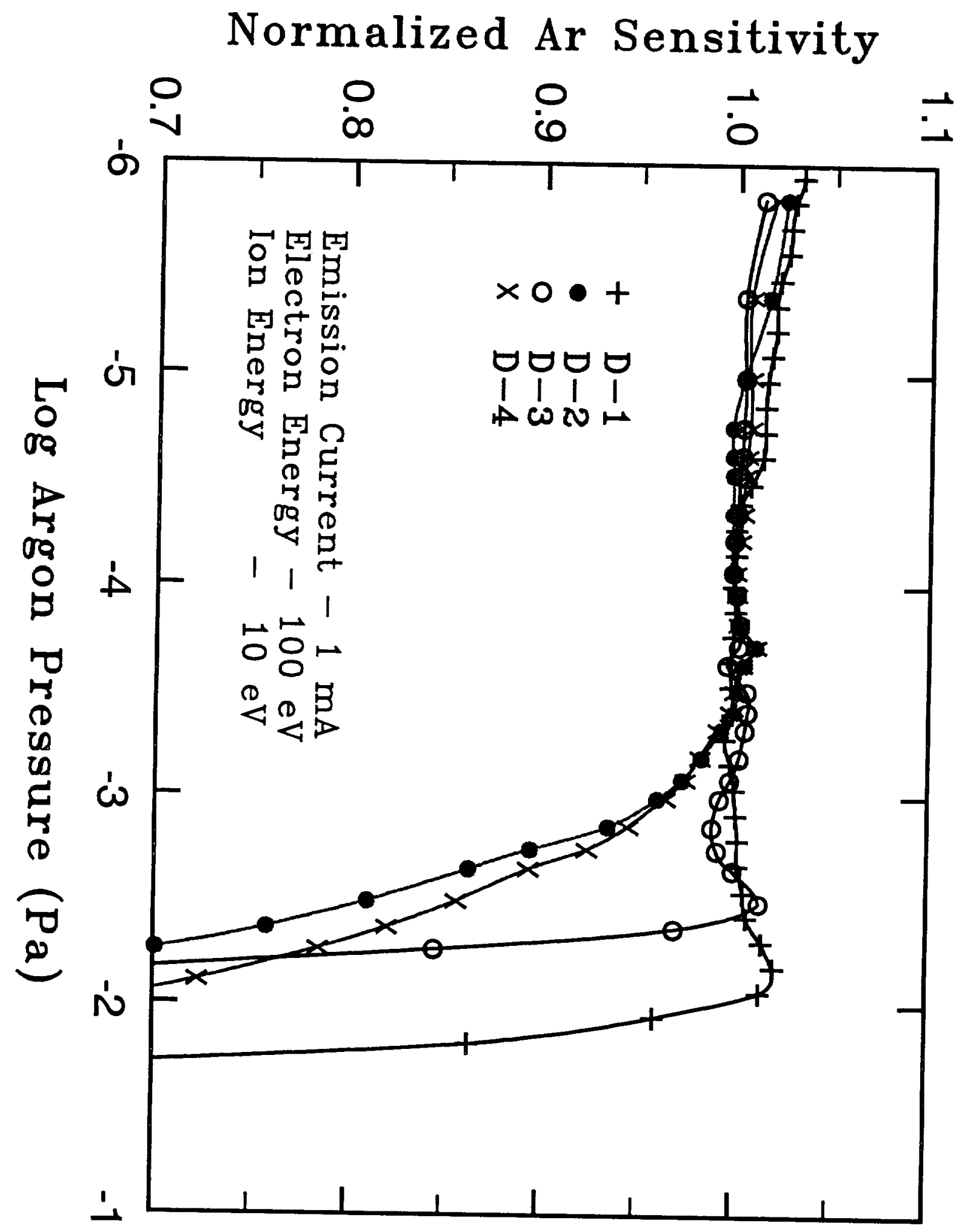


Ion Current Change, \%

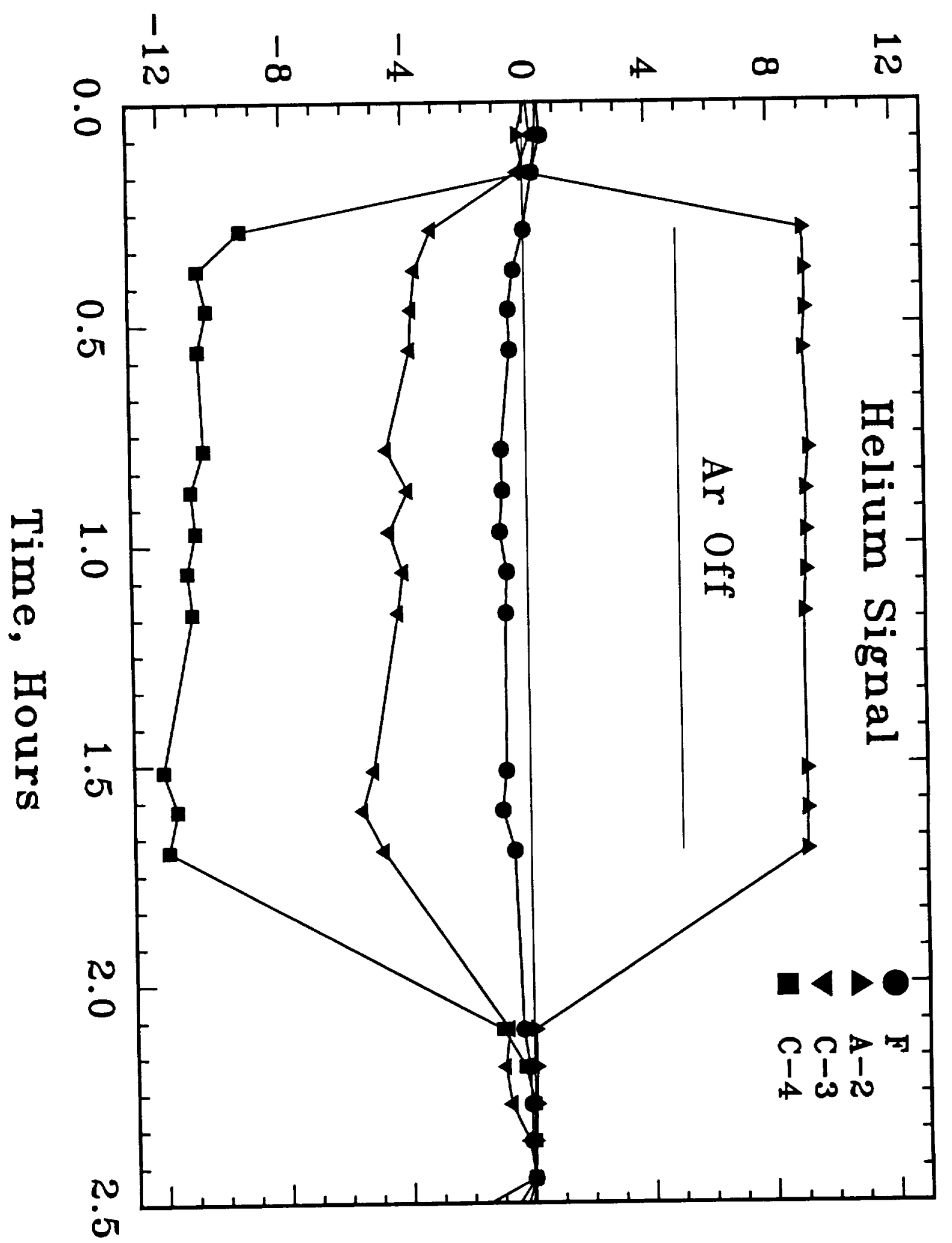




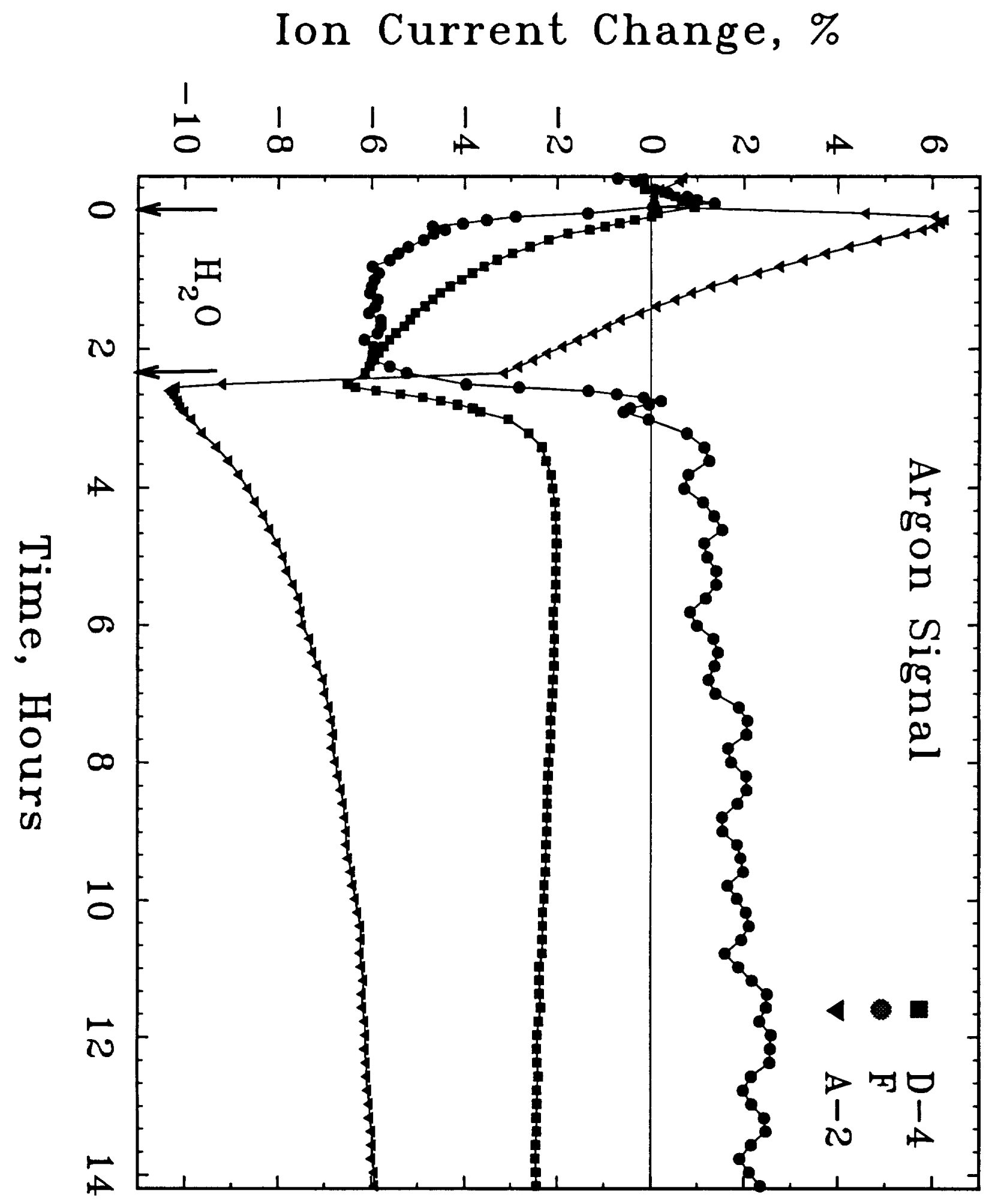


